\title{
Study of central line-associated bloodstream infections in intensive care unit: a prospective observational study
}

\author{
Rode $A^{1}$, Bansod P. $Y^{2}$, Gujela $A^{3}$, Singh $A^{4}$ \\ ${ }^{1}$ Dr. Avinash Rode, Professor, Department of Surgery, GMC, Gondia, ${ }^{2}$ Dr. Prasad Y. Bansod, Senior Resident, ${ }^{3}$ Dr. Ajit \\ Gujela, Junior Resident, ${ }^{4}$ Dr. Alok Singh, Junior Resident, ${ }^{2,3,4}$ Authors are affiliated with Department of Surgery, NKP \\ Salve Institute of Medical Sciences, Nagpur, Maharashtra, India.
}

Address for Correspondence: Dr. Prasad Y. Bansod, Email: rabbu7288@gmail.com

\begin{abstract}
Introduction: Central line associated blood stream infection (CLABSI) is a primary blood stream infection in a patient that had a central line within the 48-hour period before the development of bacteraemia and is not related to an infection at another site. It is often associated with serious infectious complications resulting in significant morbidity, increased duration of hospitalization and additional medical costs. Objective: We aimed to study the course of infection, microbiology of CLABSI, \& to identify the degrees of severity of sepsis associated with CLABSI. Materials \& Methods: Patients admitted in Intensive care units who fulfilled the inclusion criteria were enrolled. Various demographic, microbial and patients characteristics were noted along with outcome using a prestructured proforma. Results: We studied 58 patients in ICU in which males were common, Sepsis seen in $43 \%$ of patients, 37\% had Staph. Aureus, and the mortality was $24.1 \%$. Conclusion: CLABSI infection is best prevented rather than cured. It has mortality as high as $24.13 \%$. It can manifest with varying degrees of severity of sepsis. Organ Dysfunction, multi organ involvement, TLC abnormalities, Oliguria, altered mental status, Hypotension are all markers of poor prognosis. More scientific data on the subject is required to formulate guidelines and protocols for prevention and treatment of CLABSI.
\end{abstract}

Keywords: Bacteraemia, Central line, CLABSI, Central venous catheter, Sepsis, Septic shock

\section{Introduction}

Central line-associated blood stream infection (CLABSI) is a primary blood stream infection (BSI) in a patient that had a central line within the 48-hour period before the development of the BSI and is not bloodstream related to an infection at another site. However, some BSIs are secondary to other sources other than the central line (e.g., pancreatitis, mucositis) which may not be easily recognized [1-5].

Central venous catheter related bacteremia cause $\sim 14 \%$ of nosocomial infections and Central venous catheters account for most of these blood stream infections [1-3]. Central venous catheters (CVCs) are increasingly used in hospitals to manage critically ill patients requiring long term intravenous medications. Central lineassociated blood stream infection (CLABSI) occurring

Manuscript received: $26^{\text {th }}$ March 2017

Reviewed: $4^{\text {th }}$ April 2017

Author Corrected: $13^{\text {th }}$ April 2017

Accepted for Publication: $19^{\text {th }}$ April 2017 in the intensive care unit (ICU) are common, costly and potentially lethal. In the ICU, central venous access might be needed for administration of fluids, drugs, and blood products for infusion therapy, nutritional support, hemodynamic monitoring, plasmapheresis, and hemodialysis. Moreover, some catheters may be inserted in urgent situations, during which optimal attention to aseptic technique might not be feasible. CVCs have a higher infection risk than other indwelling catheters [2]. This causes significant morbidity and mortality to the critically ill patient. Central lineassociated blood stream infection (CLABSI) are considered among the first and most preventable classes of nosocomial infections [3].

Patients with CVCs are at risk of developing infectious complications like local insertion-site infection, CLABSI, septic thrombophlebitis, endocarditis and other metastatic infections. The most serious 
complications are bacteremia, sepsis and death [4]. Definitive diagnosis of central venous catheter infection is made by using a combination of clinical signs and symptoms together with the blood culture techniques. Subclavian vein CVC's are associated with lower CLABSI rates than internal jugular CVC's, whereas femoral CVC's have highest rates of CLABSI [5].

Potential risk factors for CLABSI include underlying disease, method of insertion, site, duration, and purpose of catheterization. Local risk factors like poor personal hygiene, occlusive transparent dressing, and moisture around the exit site. S. aureus nasal colonization, and contiguous infections support the role of bacterial colonization in the pathogenesis. Other risk factors include contamination, inadequate water treatment, dialyzer re-use, older age, higher total intravenous iron dose, increased recombinant human erythropoietin dose, lower hemoglobin level, lower serum albumin level, diabetes mellitus, peripheral atherosclerosis, and recent hospitalization or surgery [6].

Central catheter is often associated with serious infectious complications; CLABSI can cause endocarditis possibly in up to $23 \%$ of patients or more [7-10]. So far, there are very few studies that have been conducted on CLABSI in India. This study was undertaken to study the CVC-related infections in the ICU and to identify the factors influencing it, which would help to institute better prophylactic measures.

\section{Materials and Methods}

This prospective observational study was conducted at 10 bedded Intensive Care Unit at GMC Gondia, Maharashtra, India. Patients admitted in ICU and in whom CVC was inserted after ICU admission were included in the study. Duration of the study was during the period from January 2016 to January 2017. The research study was done according to the ethical principles laid down by the Helsinki declaration and permission from the hospital ethics committee was taken. Cases were described as per clinical entity, having bacteremia, fungemia ( $+/-$ clinical sepsis) because of an indwelling Central vascular Catheter with no other apparent source of infection. Two blood samples were collected after 48 hours of central line insertion (one obtained from the lumen of the central catheter/ endoluminal swab/catheter tip and the second from a peripheral venipuncture). If both the samples had same organism growth on culture media, it was termed a positive case.

Uncomplicated cases are those in which there is laboratory evidence of presence of bacteria in blood by the growth of same organism in peripheral and CVC blood cultures but without any signs of inflammation. Sepsis is absent, while complicated case is CLABSI plus varying degrees of severity of sepsis. Sepsis according to degrees of severity is classified as a) Sepsis b) Severe Sepsis and c) Septic Shock.

\section{a) Sepsis}

The sepsis was defined as two or more of the symptoms (mandatory),

- Hypothermia (temperature $<96^{0} \mathrm{~F}$ ) or fever (temperature $>100.9^{0}$ ),

- Tachycardia (heart rate more than $90 / \mathrm{min}$ ),

- Tachypnea (respiratory rate $>20 / \mathrm{min}$ ),

- Leucocytes greater than 12000 cells $/ \mathrm{mm}^{3}$ or less than 4000 cells $/ \mathrm{mm}^{3}$ and documented infection.

\section{b) Severe sepsis}

Sepsis and Organ dysfunction

- hypotension ( blood pressure $<90 \mathrm{mmHg}$ or decrease of $>40 \mathrm{mmHg}$ ) or

- organ hypo perfusion, including

- Metabolic acidosis

- Oliguria (urine output $<400 \mathrm{ml} / 24$ hrs. despite adequate fluid treatment) or

- Acute alteration of consciousness

\section{c) Septic shock}

- Hypotension (despite adequate fluid treatment) and

- Hypo perfusion of organs

Fluid resuscitation was considered adequate when CVP $>10 \mathrm{cms}$ of water

\section{Definitions of Positive findings taken into study}

- Tachycardia- Heart rate more than 90 beats per minute

- Fever- Body temperature more than $100.9{ }^{0} \mathrm{~F}$.

- Tachypnea- Respiratory rate of more than 20 /minute

- Oliguria- Urine output of less than $400 \mathrm{ml}$ in 24 hours.

- Hypothermia- body temperature of lesser than $96.8^{0} \mathrm{~F}$

- Hypertension- blood pressure of more than 130/90 $\mathrm{mmHg}$

- Bradycardia- Heart rate less than 60 beats per minute. 
All patients enrolled in the study were followed daily for the development of new onset sepsis 48 hours after the insertion of the CVC. Clinical and hematological parameters were obtained every alternate day or earlier if sepsis was suspected. New onset sepsis was suspected when two or more of the following conditions were present along with suspicion of the infection

In the setting of local or systemic sepsis, a set of blood cultures was obtained both percutaneously from a single venipuncture site, as well as through the catheter. In the absence of local or systemic sepsis, a set of blood cultures was routinely taken from the catheter at the time of catheter removal. Each collected set of blood culture bottles consisted of an aerobic BancTec® bottle, an anaerobic BancTec ${ }^{\circledR}$ bottle and a fungal blood culture tube. Blood cultures were processed at $35^{\circ} \mathrm{C}$ for 7 days by the BACTEC-460® radiometric method. Fungal blood cultures were processed using conventional methods. Seldinger technique was used for central catheter insertion.

\section{Inclusion criteria}

1. Consenting Patients/relatives in age group 18-60 years with both genders.

2. Patients with Central venous catheter inserted as per defined institute asepsis antisepsis protocols at ICU.

3. Patients satisfying the case definition criterion as enumerated.

\section{Exclusion Criteria}

1. Non consenting patients/relatives.

2. Patients with other obvious source of infection.

3. Immune-suppressed states.

4. Patients developing CVC insertion procedure related complications e.g. Hematoma, pneumothorax etc.

5. Patients with PICC lines, Tunneled catheters, totally implanted catheters.

Statistical Analysis- For interpretation of observations made as categorical data. A two-tailed $P$ value of $<0.05$ was considered statistically significant. All statistical computations were performed using the SPSS software.

\section{Results}

In present study, a total of 58 patients were studied. Out of them 36 (i.e. 62.06\%) were male and 22 (37.94\%) were female. Male to female's ratio was 1.6:1. The commonest age group affected was 41-50 years (19 patients i.e. 32.7\%). The mean age of affected patients was 42.03 years with a standard deviation of 11.32. The youngest patient was 22 years old while the oldest was 59 years old. (Fig. 1)

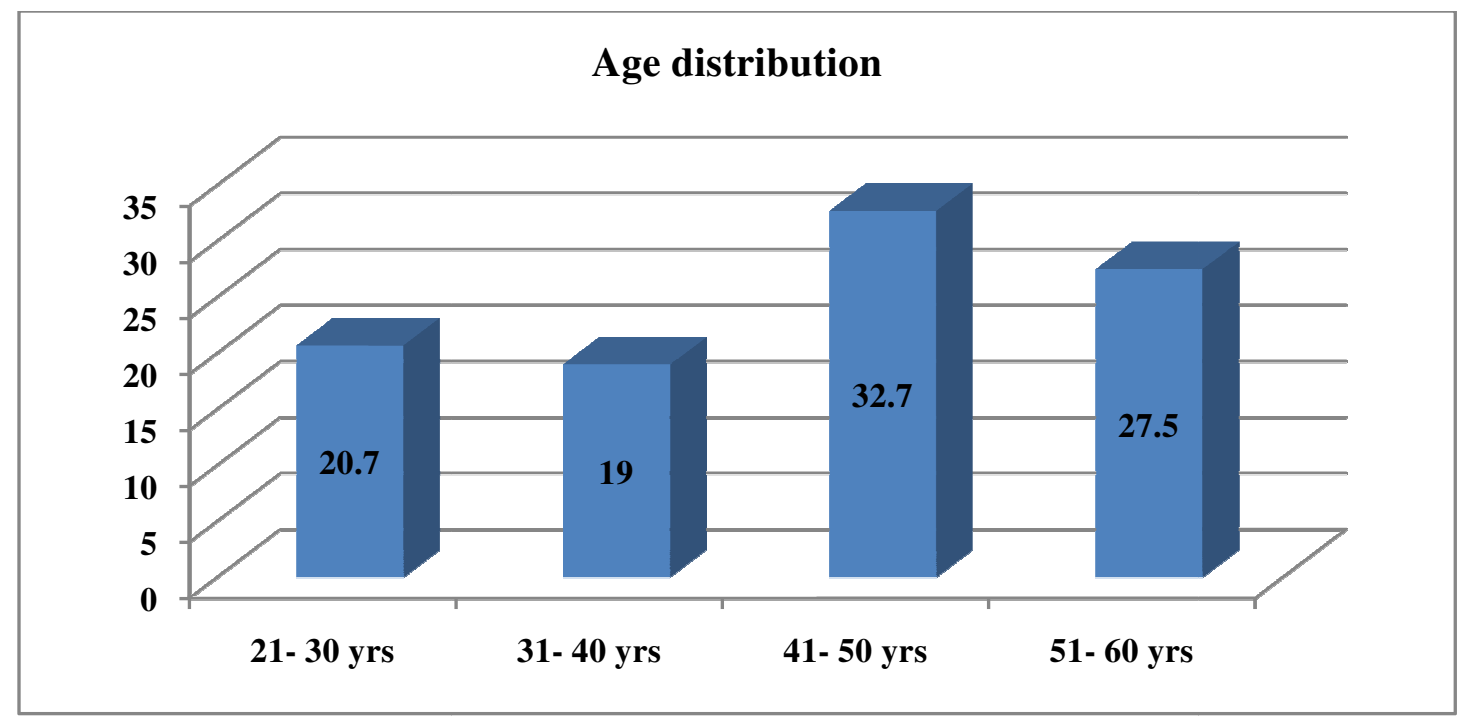

Fig.-1: Showing age distribution

Out of total 58 patients developing CLABSI, 5 patients $(8.62 \%)$ had bacteremia without obvious signs of sepsis, while the rest 53 patients $(91.38 \%)$ developed frank sepsis. All cases with frank sepsis were further categorized based on severity of sepsis (i.e. Sepsis v/s Severe Sepsis v/s Septic Shock). Out of 53 patients who developed sepsis, 25 patients $(47.16 \%)$ had mild sepsis, 20 patients (37.73\%) progressed to developed severe sepsis, and 8 patients $(15.09 \%)$ developed septic shock. (Fig. 2) 


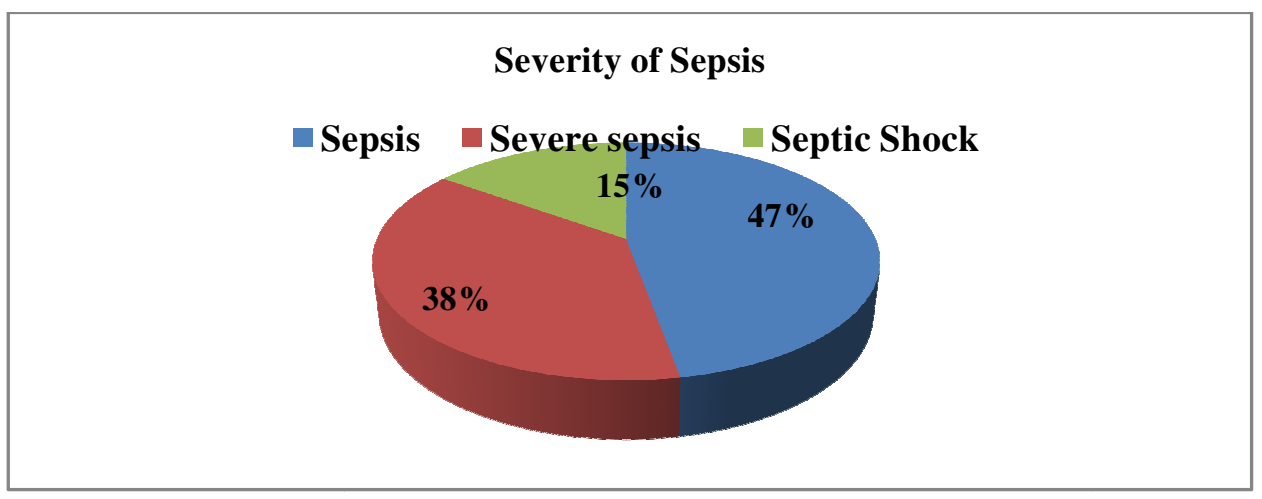

Fig.-2: Showing Severity of Sepsis

The most common indication for insertion of CVC was Monitoring of Central venous pressure (in 32 patients i.e. $55.17 \%$ ). There were multiple indications for CVC insertion in some patients. It was an indication in 25 patients (43.10\%) Prompt fluid resuscitation was an indication in 11 patients (18.96\%). In 10 patients (i.e. 17.24\%) CVC in form of HD catheter was required for giving Hemodialysis. (Table 1)

Table-1: Showing distribution of patients according to Indication of CVC insertion.

\begin{tabular}{|c|c|c|}
\hline Indication of CVC insertion & Number of patients & Percentage (\%) \\
\hline CVP Monitoring & 32 & 55.17 \\
\hline Central Venous Access & 25 & 43.10 \\
\hline Fluid Resuscitation & 11 & 18.96 \\
\hline Hemodialysis & 10 & 17.24 \\
\hline
\end{tabular}

Fever was the most common symptom present in 54 patients i.e. $93.10 \%$. The next most common sign was Tachycardia in 45. This was followed by Tachypnea in 41 patients, $70.68 \%$.

Symptoms present in frequencies less than $50 \%$ but otherwise found to be in higher frequencies in septic shock were oliguria, altered mental status and Hypothermia (Fig. 3)

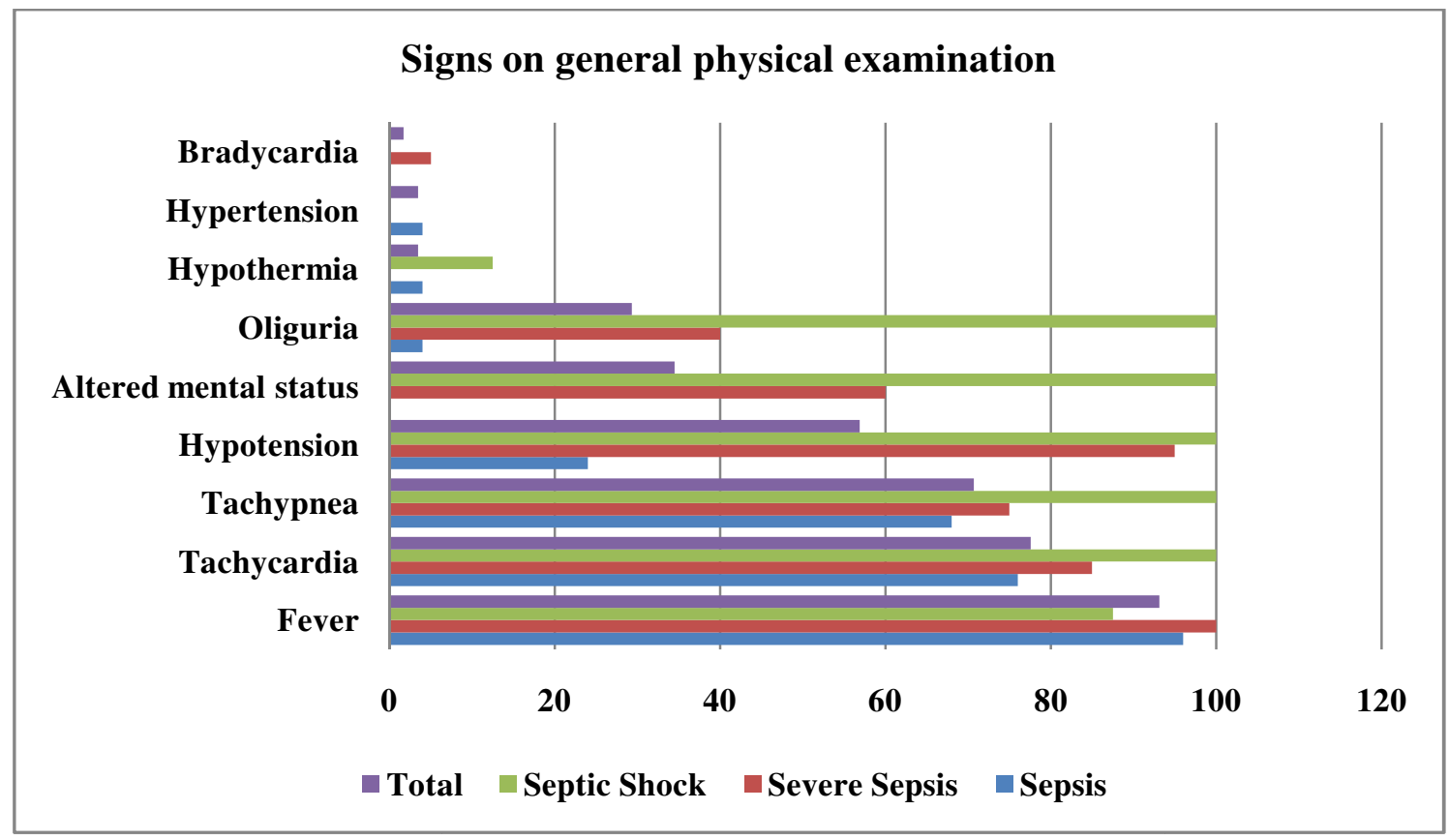

Fig.-3: Showing Distribution of signs on physical examination 
The local signs of inflammation were present in 13 patients i.e. $22 \%$ and absent in 45 patients i.e. $78 \%$.

Central venous access was achieved through Internal Jugular Vein cannulation in 27 patients (46.55\%), through femoral vein cannulation in 22 patients $(37.93 \%)$ and through subclavian vein cannulation in 9 patients $(15.51 \%)$. (Table 2$)$

Table-2: Showing Site of central venous cannulation.

\begin{tabular}{|c|c|c|}
\hline $\begin{array}{c}\text { Site of Central Venous } \\
\text { Cannulation }\end{array}$ & $\begin{array}{c}\text { Number of patients } \\
(\mathbf{n = 5 8})\end{array}$ & Percentage (\%) \\
\hline Internal Jugular Vein & 27 & 46.55 \\
\hline Femoral vein & 22 & 37.93 \\
\hline Subclavian Vein & 9 & 15.51 \\
\hline Total & $\mathbf{5 8}$ & $\mathbf{1 0 0}$ \\
\hline
\end{tabular}

Number of patients having CVC in-situ for more than 12 days were 45 i.e. $77.58 \%$ while 13 patients i.e.22.42\% had CVC in-situ for less than 12 days. (Table 3)

Table-3: Showing distribution of patients according to number of catheter days.

\begin{tabular}{|c|c|c|c|c|c|c|c|c|}
\hline $\begin{array}{c}\text { Number of } \\
\text { Catheter Days }\end{array}$ & \multicolumn{2}{|c|}{$\begin{array}{c}\text { Sepsis } \\
\text { N=25 }\end{array}$} & \multicolumn{2}{c|}{$\begin{array}{c}\text { Severe Sepsis } \\
\text { N=20 }\end{array}$} & \multicolumn{2}{c|}{$\begin{array}{c}\text { Septic Shock } \\
\text { N=8 }\end{array}$} & \multicolumn{2}{c|}{ Total N=58 } \\
\cline { 2 - 10 } & No & $\mathbf{( \% )}$ & No & $\mathbf{( \% )}$ & No & $(\mathbf{\%})$ & No & $(\%)$ \\
\hline$<12$ days & 4 & 16 & 4 & 20 & 0 & 0 & 13 & 22.42 \\
\hline$>12$ days & 21 & 84 & 16 & 80 & 8 & 100 & 45 & 77.58 \\
\hline
\end{tabular}

Most common organism cultured was Staphylococcus aureus (isolated in 22 patients i.e.37.94\%) 6 patients out of 22 succumbed to infection due to it)

Pseudomonas aeruginosa was next in the list affecting 11 patient i.e.18.96\% of total cases. .It was the organism with second highest case fatality (i.e. 5 patients out of 11 succumbed to infection). Klebsiella Pneumoniae was isolated in 4 patient i.e.6.89\% of cases. Escherichia coli was isolated in 5 patients, $8.62 \%$ of all cases. The candida group was isolated from 7 patient i.e. $12.07 \%$ of all cases but had zero mortality. Coagulase negative staphylococci were cultured in 6 patients i.e. $10.35 \%$ of all cases .It also didn't had any associated mortality. Acinetobacter baumannii was isolated from 3 patients $(5.17 \%)$. (Fig.4)

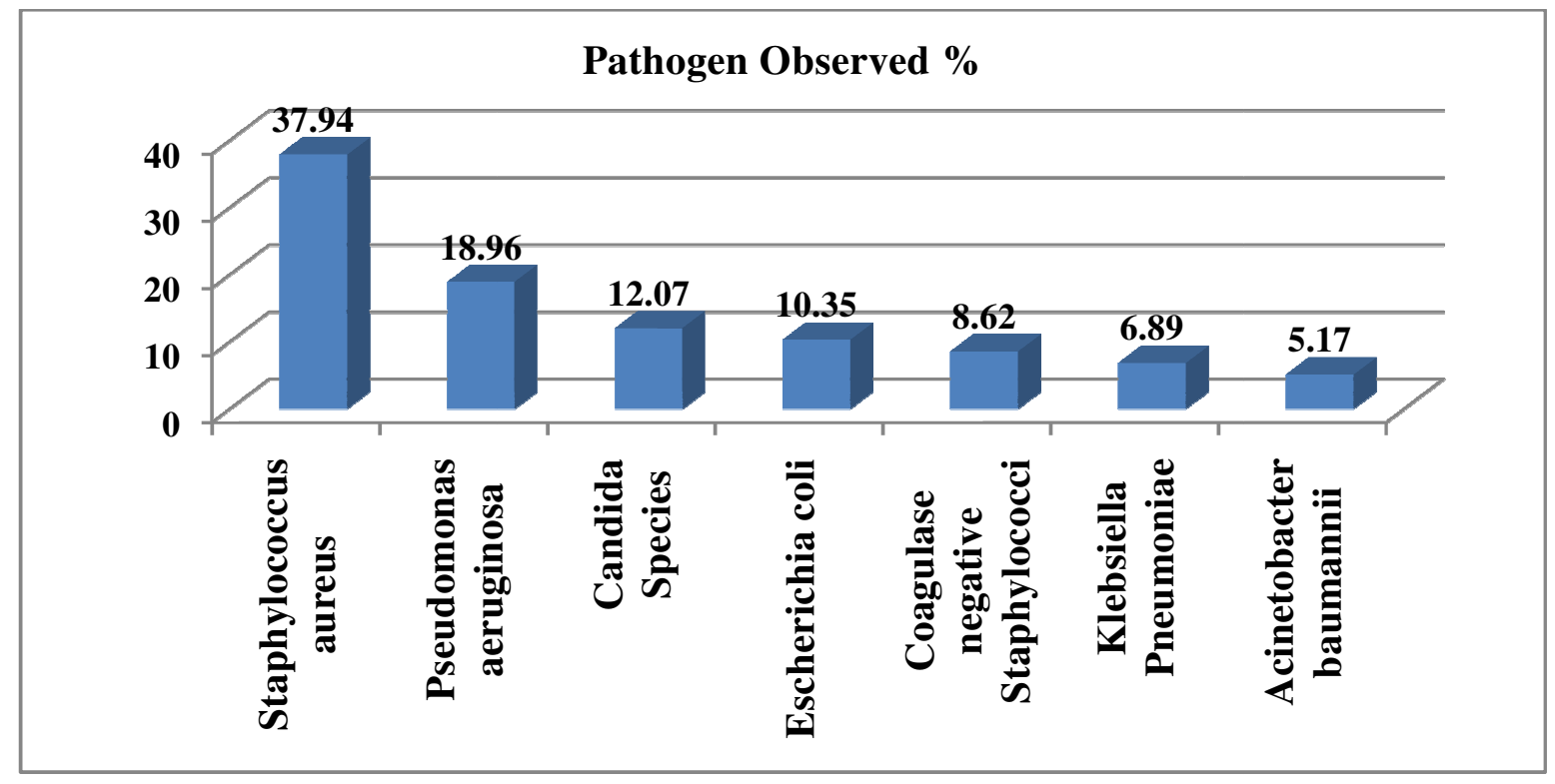

Fig.-4: Showing distribution of patients according to pathogen observed 
Of 58 patients developing CLABSI 14 patients i.e. $24.13 \%$ succumbed to infection and the rest 44 patients i.e. $75.86 \%$ recovered with treatment. (Table 4 \& Fig. 5)

Table-4: Showing distribution of Mortality in different categories of patients.

\begin{tabular}{|c|c|c|c|c|c|c|c|c|c|c|}
\hline \multirow[t]{3}{*}{ Outcome } & \multicolumn{6}{|c|}{ Complicated CLABSI } & \multirow{2}{*}{\multicolumn{2}{|c|}{$\begin{array}{c}\text { Uncomplicated } \\
\text { CLABSI } \\
\text { N=5 }\end{array}$}} & \multirow{2}{*}{\multicolumn{2}{|c|}{$\begin{array}{l}\text { Total } \\
\mathrm{N}=58\end{array}$}} \\
\hline & \multicolumn{2}{|c|}{$\begin{array}{l}\text { Sepsis } \\
N=25\end{array}$} & \multicolumn{2}{|c|}{$\begin{array}{c}\text { Severe sepsis } \\
\quad \mathrm{N}=\mathbf{2 0}\end{array}$} & \multicolumn{2}{|c|}{$\begin{array}{c}\text { Septic shock } \\
\text { N=8 }\end{array}$} & & & & \\
\hline & No & $\%$ & No & $\%$ & NO & $\%$ & NO & $\%$ & NO & $\%$ \\
\hline Recovery & 25 & 100 & 13 & 65 & 1 & 12.5 & 5 & 100 & 44 & 75.86 \\
\hline Death & 0 & 0 & 7 & 35 & 7 & 87.5 & 0 & 0 & 14 & 24.13 \\
\hline
\end{tabular}

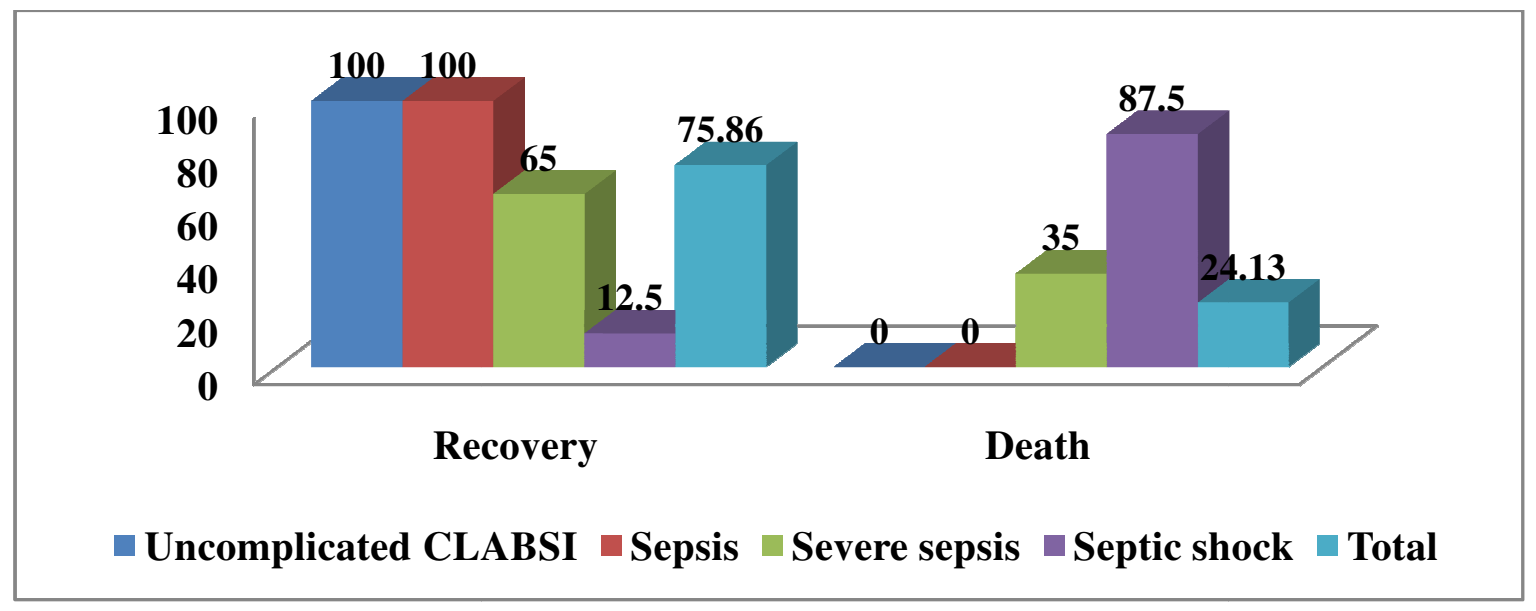

Fig.-5: Showing distribution of Mortality in different categories of patients

\section{Discussion}

This study was designed to assess the course of infection, microbiology of CLABSI, \& to identify the degrees of severity of sepsis. We also evaluated differences in clinical signs observed, sites, duration of central line and mortality. A total of 58 patients were evaluated during the course of this study.

Most common age group affected was found to be 4150 years i.e. $32.75 \%$, the second most common age group affected was 51-60 years (16 patients i.e. $27.5 \%$ ). The mean age of affected patients was 42 years, this observation was in accordance to a study by Mervyn Mer et al [11], and Johnson et al [12] showed median age of 47 years.

In the present study out of a total of 58 patients 36 patients (i.e. 62.06\%) were males and 22patients $(37.94 \%)$ were females. The male to female's ratio was 1.6:1. This finding was also comparable to the study by Mervyn Mer et al [11], Johnson et al [12] in his study had male proportion of $61 \%$ and females $39 \%$ with a male: female ratio of $1.56: 1$.
Of total 58 patients developing CLABSI, 5 patients $(8.62 \%)$ had bacteremia without obvious signs of sepsis, while the rest 53 patients $(91.38 \%$ ) developed CLABSI with varying degrees of severity of sepsis. This finding was in accordance with the study of Harsha V Patil et al [13] in which $7.41 \%$ of patients were having Central line related bacteremia and rest $92.59 \%$ had CLABSI with sepsis. Patients developing CLABSI were categorized into sepsis, severe sepsis, and septic shock as per case definitions, Out of 53 patients who developed sepsis, 25 patients $(47.16 \%)$ had mild sepsis and 20 patients $(37.73 \%)$ progressed to developed severe sepsis. Further 08 patients $(15.09 \%)$ developed septic shock.

Indications for CVC insertion in present study were CVP monitoring in 32 patients, venous access in 25 patients, fluid resuscitation in 11 patients \& hemodialysis in 10 patients. On comparing it with literature similar findings noted by Sanjeev Sinha et al [14] et al in which the occurrence of CLABSI was $83 \%$ when central line was inserted for Monitoring of central 
venous pressure, which in this study would account to 74.13\% (CVP monitoring 55.17\%+fluid resuscitation $18.96 \%$ ) and was $17 \%$ when the indication was for gaining central venous access which in present study was $25.10 \%$.

In general physical exam findings the most common positive finding was fever in 54 patients, tachycardia in 45 patients. 13 patients out of 58 i.e. $22.41 \%$ had positive local signs of inflammation while the rest 45 patients did not had any local inflammatory signs at the site of insertion. The finding was similar to the observation made in one study by Patil HV et al [15], in which local signs were present in 13 patients out of total 54 patients (i.e. $31.70 \%$ ) while the rest 41 out of 54 i.e. $75.92 \%$ had no local signs of inflammation.

The occurrence of CLABSI according the site of insertion of CVC was studied for IJV, femoral vein and subclavian vein, in this study and was found to be $46.55 \%, 37.39 \%$ and $15.51 \%$ respectively.

These findings were in contrast to findings of O'Connor A et al [16] in which the incidence of CLABSI in IJV was $68 \%$, in femoral vein was $6.5 \%$ and in subclavian vein was $24.6 \%$. These differences were attributed mainly to differential preference of central cannulation sites which varied across different institute protocols.

In this study the number of patients having CVC in-situ for more than 12 days were 45 i.e. $77.58 \%$ while rest 13 patients i.e. $22.42 \%$ had CVC in-situ for less than 12 days. This result was comparable to the findings made by Parameswaran et al [17] who demonstrated that the risk of infection for catheters placed for more than 12 days was 2.21 times higher than that of those who had CVC in situ for less than 12 days.

The microbiology of CLABSI in this study showed Staphylococcus aureus as most common organism cultured in $37.94 \%$ followed by Pseudomonas in $18.96 \%$ and Candida species in $12.07 \%$. Coagulase negative Staphylococcus was reported in $10.35 \%$, Klebsiella pneumonia in $6.89 \%$, Escherichia coli in $8.62 \%$, and Acinetobacter baumannii in $5.17 \%$ In a study by Chopdekar K et all [18], the incidence of Staphylococcus aureus was $13.2 \%$ (divided as MRSA $11.32 \%+$ MSSA $1.88 \%$ ), Pseudomonas was seen in $16.95 \%$, candida species in $22.64 \%$, coagulase negative Staphylococcus in $11.32 \%$, Klebsiella pneumoniae in $9.43 \%$, Escherichia coli in $3.77 \%$ patients,
Acinetobacter baumannii in $1.88 \%$. This study however in addition isolated other organism which included E.faecalis, Proteus vulgaris and Citrobacter koseri. Study by Parameswaran et al [17] found Staphylococcus aureus in $40 \%$, candida species and Pseudomonas aeruginosa in $16 \%$, coagulase negative staphylococcus, Escherichia coli and klebsiella pneumoniae in $8 \%$ each and Acinetobacter baumannii in $4 \%$ of patients. Our results were at times in concordance with few studies and at times in contrast to other studies.

These differences were mainly due to differences of antibiogram and isolates across various sites of studies and the differences in their infection management protocols and antibiotic policies

The overall mortality observed in our study was $24.13 \%$ (i.e. 14 of 58 patients succumbed to infection) and in terms of severity of sepsis mortality was $35 \%$ in severe sepsis and $87.5 \%$ in septic shock. In a study by Chopdekar et al [18] the mortality associated with CLABSI was found to be $33.3 \%$ which was comparable to the results of this study.

Limitations of his study do exist as this was a single site study with a small sample size of 58 patients. Given the geographical concentration of the study population, small sample size, convenience, and homogeneity of the sample the generalizability of this study and its results is hence limited and may be flawed.

The antibiotic resistance and sensitivity patterns were varied in all isolates even amongst the same organism cultured. Hence antibiotic treatment was not investigated in this study. Given institute protocol preferences for site and type of Catheters the results under corresponding subheadings may have a selection bias confounding the observations and results hence inferred.

\section{Conclusion}

CLABSI is an infection which is best prevented rather than cured because of high associated mortality rates which were as high as $24.13 \%$ in the present study. It can manifest with varying degrees of severity of sepsis. Organ Dysfunction, multi organ involvement, TLC abnormalities, Oliguria, altered mental status, Hypotension are all markers of poor prognosis. The data and observations presented in this study are important and offer direction for the use of CVCs in critically ill 
patients, irrespective of location, particularly in light of the many controversies that exist, and also in view of the suggestions by various workers in the field. However more scientific data on the subject is required to formulate guidelines and protocols for prevention and treatment of CLABSI.

\section{Funding: Nil, Conflict of interest: None Permission of IRB: Yes}

\section{References}

1. Longo DL, Fauci AS, Kasper DL, Hauser SL, Jameson JL, Loscalzo J: Harrisons principles of internal medicine. 18 ed. New York. The McGraw-Hill Companies, Inc. 2011, p1116.

2. O'Grady NP, Alexander M, Burns LA, Dellinger EP, Garland J, Heard SO, Lipsett PA, Masur H, Mermel LA, Pearson ML, Raad II, Randolph AG, Rupp ME, Saint S; Healthcare Infection Control Practices Advisory Committee (HICPAC). Guidelines for the prevention of intravascular catheter-related infections. Clin Infect Dis. 2011 May;52(9):e162-93. doi: 10.1093 /cid/ cir257. Epub 2011 Apr 1.

3. Longo DL, Fauci AS, Kasper DL, Hauser SL, Jameson JL, Loscalzo J. Harrisons principles of internal medicine. 18 ed. New York. The McGraw-Hill Companies, Inc.2011 p1117.

4. Naomi P. O'Grady, Didier P. Catheter-Related Infections in the Critically Ill. Norvell. Kluwer Academic Publishers, 2004, p90.

5. Foster C, Mistry N, Peddi P, Sharma S.The Washington Manual of Medical Therapeutics. 33rd ed. New Delhi:WoltersKluver (India) Pvt Ltd. 2010, p503.

6. Jordi R, Martin H, Emilio D, Alejandro R. Infectious Diseases in Critical Care, Berlin, Springer Science \& Business Media, 2010, p7.

7. Rijnders B, Intravascular Catheter-Related Infections: A Clinical Focus on Prevention, Diagnosis \& Treatment.Belgium, Leuven University Press, 2003, p 21.

8. Dimick JB, Pelz RK, Consunji R, Swoboda SM, Hendrix CW, Lipsett PA. Increased resource use associated with catheter-related bloodstream infection in the surgical intensive care unit. Arch Surg. 2001 Feb;136(2):229-34.

9. World Health Organization (WHO) Preventing bloodstream infections from central line venous catheters [Internet].(cited 28august 2014)Available from:http://www.who.int/patientsafety/implementation/ bsi/en/

10. Esherick J, The Tarascon Clinical Review Series: Internal Medicine, Chapter 48- Management of Catheter Related Blood stream infections, Jones \& Bartlett Publishers, 2012p217

11. Mer M. Intravascular catheter-related infectioncurrent concepts. Southern African Journal of Critical Care. 2006; 22(1):4-12.

12. Mermel LA, Farr BM, Sherertz RJ, Raad II, O'Grady N, Harris JS, Craven DE; Infectious Diseases Society of America, American College of Critical Care Medicine, Society for Healthcare Epidemiology of America. Guidelines for the management of intravascular catheter-related infections. J Intraven Nurs. 2001 May-Jun;24(3):180-205.

13. Patil HV, Patil VC, Ramteerthkar MN, Kulkarni RD. Central venous catheter-related bloodstream infections in the intensive care unit. Indian J Crit Care Med. 2011 Oct;15(4):213-23. doi: 10.4103/0972-5229. 92074.

14. Deepti, Sinha S, Sharma SK, Aggarwal P, Biswas A, Sood S, Ragunandan P, Ekka M, Xess I, Sreenivas V. Central venous catheter related bloodstream infections in medical intensive care unit patients in a tertiary referral centre. Indian J Chest Dis Allied Sci. 2014 Apr-Jun;56(2):85-91.

15. Patil HV, Patil VC, Ramteerthkar MN, Kulkarni RD. Central venous catheter-related bloodstream infections in the intensive care unit. Indian J Crit Care Med. 2011 Oct;15(4):213-23. doi: 10.4103/0972-5229. 92074.

16. O'Connor A, Hanly AM, Francis E, Keane N, McNamara DA. Catheter associated blood stream infections in patients receiving parenteral nutrition: a prospective study of 850 patients. Journal of clinical medicine research. 2013 Jan 11;5(1):18-21. 
17. Parameswaran R, Sherchan JB, Varma M, Mukhopadhyay C, Vidyasagar S. Intravascular catheterrelated infections in an Indian tertiary care hospital. The Journal of Infection in Developing Countries. 2010 Nov $11 ; 5(06): 452-8$.
18. Chopdekar K, Chande C, Chavan S, Veer P, Wabale V, Vishwakarma K, Joshi A. Central venous catheterrelated blood stream infection rate in critical care units in a tertiary care, teaching hospital in Mumbai. Indian $\mathbf{J}$ Med Microbiol. 2011 Apr-Jun; 29(2):169-71. doi: 10. 4103/0255-0857.81796.

\section{How to cite this article?}

Rode A, Bansod P.Y, Gujela A, Singh A. Study of central line-associated bloodstream infections in intensive care unit: a prospective observational study. Int J Med Res Rev 2017;5(04):429- 437. doi:10.17511/ijmrr. 2017.i04.09. 